\title{
Cientintifica
}

http://revistas.udistrital.edu.co/ojs/index.php/revcie/index

ARTí́CULO DE INVESTIGACIÓN

\section{Transformación de archivos DICOM a formatos XML, JPEG y PNG}

\author{
Converting DICOM filles to XML, JPEG and PNG Formats
}

Transformando DICOM para arquivos XML, JPEG e PNG

\author{
Omar Hernando Moreno Torres ${ }^{1}$ \\ Juan Sebastián González Sanabria² \\ Javier Antonio Ballesteros Ricaurte ${ }^{3}$
}

Fecha de recepción: octubre 2014

Fecha de aceptación: noviembre 2014

Para citar este artículo: Moreno, O., González, J. y Ballesteros J. (2005). Transformación de archivos DICOM a formatos XML, JPEG y PNG. Revista Científica, 21, 71-80. Doi: 10.14483/udistrital.jour.RC.2015.21.a7

\section{Resumen}

Se presenta un modelo para la extracción de información contenida en los objetos de los archivos de tipo DICOM y su correspondiente transformación a archivos de tipo XML, bajo el estándar CDA de HL7. Se inicia con el análisis de la estructura de los archivos de tipo DICOM y de una serie de librerías que interactúan con la información requerida, para posteriormente presentar el modelo de transformación y el esquema resultante de archivo XML. En la parte final se plantea una transformación a imágenes no diagnósticas en formatos JPG y PNG.

Palabras Clave: dcm4che2, DICOM, XML.

\section{Abstract}

There is shown a model for extracting data from objects in DICOM files and how to convert those into XML files format, with a HL7 CDA standard. Starting with the DICOM files structure analysis and the study of a set of libraries that interact with the requested information, to show then the converting model and the resulting XML file scheme. Finally, there is raised the conversion to undiagnosed images in JPG and PNG formats.

Keywords: dcm4che2, DICOM, XML.

\section{Resumo}

No artigo de um modelo para a extração de informações contidas nos arquivos de objetos tipo DICOM e seus arquivos tipo XML de transformação correspondente sob o padrão CDA de HL7. Ele começa com a análise da estrutura de arquivos tipo DICOM e um conjunto de bibliotecas que interagem com as informações necessárias, em seguida, apresentar a reorganização eo arquivo do esquema XML resultante. No final de uma transformação não imagens de diagnóstico em formato JPG e PNG.

Palavras-chave: dcm4che2, DICOM, XML.

Universidad Pedagógica y Tecnológica de Colombia, Tunja-Colombia. Contacto: omar.moreno@uptc.edu.co Universidad Pedagógica y Tecnológica de Colombia, Tunja, Colombia, javier.ballesteros@uptc.edu.co

Universidad Pedagógica y Tecnológica de Colombia, Tunja-Colombia. Contacto: juansebastian.gonzalez@uptc.edu.co 


\section{Introducción}

En el ámbito médico existen más de 900 dispositivos y elementos de diferentes fabricantes involucrados en el desarrollo de procedimientos médicos en las diversas entidades prestadoras de servicios de salud, los cuales son principalmente concebidos para la generación de imágenes y análisis de información, lo cual, junto a un gran número de sistemas información utilizados por los entes organizacionales del sector salud, implica tener deficiencias respecto a la comunicación y transmisión de información, por lo que se requiere un gran esfuerzo en la implementación de estándares y homogeneización de esta información.

DICOM (Digital Imaging and Communication in Medicine) surge como un estándar para la comunicación de imágenes en medicina, con la finalidad de facilitar el intercambio de información médica utilizada por los diferentes especialistas, centros de investigación o entidades prestadoras de servicios de salud (Pianykh, 2008). Dicho formato se puede definir como una interfaz común para cualquier dispositivo de imágenes (ecógrafos, tomógrafos, rayos $x$, entre otros) en un marco estandarizado de comunicación entre ámbitos de transmisión, tratamiento o impresión.

El presente trabajo expone un modelo para la lectura y transformación de la información en archivos XML y formatos de imagen convencional (JPG y $\mathrm{PNG}$ ) contenida en las imágenes de tipo DICOM.

\section{Archivos DICOM}

DICOM (Herranz, 2003) es un estándar de formatos de archivos y de especificaciones de datos primordiales de un paciente para describir un lenguaje común entre distintos sistemas médicos (Grupo PAS, 2010). En la Figura 1 se representa el modelo de protocolo de comunicaciones DICOM, los niveles de comunicación de información representados sobre la máquina o dispositivo biomédico, a través del modelo OSI o el modelo TCP/IP, dependiendo de las circunstancias, marcas de los dispositivos, tipo y procedimiento médico al cual esté vinculado (Rosslyn, 2004).

\begin{tabular}{|c|c|c|}
\hline \multicolumn{3}{|c|}{ Aplicación Gráfica Médica } \\
\hline \multicolumn{3}{|c|}{ Intercambio de Mensajes DICOM } \\
\hline \multirow{2}{*}{$\begin{array}{c}\text { Sesión, } \\
\text { Transporte y } \\
\text { Red }\end{array}$} & $\begin{array}{l}\text { Protocolo } \\
\text { Superior de } \\
\text { DICOM para }\end{array}$ & Presentación \\
\cline { 2 - 3 } & TCP/IP & Transporte \\
\cline { 2 - 3 } & TCP & Red \\
\hline Datos & IP & Datos \\
\hline Físico & Físico \\
\hline DICOM & TCP/IP & OSI \\
\hline
\end{tabular}

Figura 1. Modelo de protocolo de comunicación DICOM Fuente: adaptado de Herranz (2003)

Este modelo define la estructura de un sistema RIS (Radiology Information System) o un PACS (Picture Archiving and Communication System) (Parisot, 2003), cuya base de funcionalidad se divide en dos componentes: el primero se relaciona con el formato y la estructura del archivo DICOM; y el segundo afina un protocolo de intercambio de datos no propietario para imágenes biomédicas e información relacionada con las mismas.

Por otra parte, la Figura 2 expone la estructura de los archivos de tipo DICOM, la cual se divide en dos partes principales: un encabezado (Header), en donde se contiene cierta información relevante del paciente; y un cuerpo (Data set), compuesto de un gran número de elementos (tags) que proporciona una información en específico.

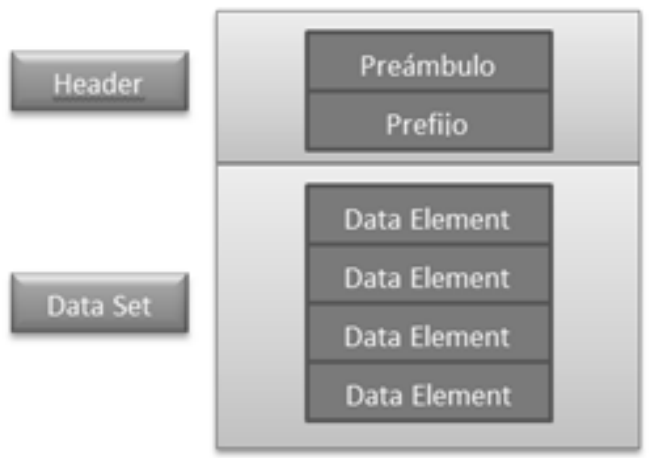

Figura 2. Estructura de un archivo DICOM Fuente: adaptado de Grupo PAS (2010) 
Los objetos de tipo IOD (Information Object Definition) se soportan en un lenguaje orientado a objetos para definir la información de imágenes biomédicas, que desde el punto de vista del paradigma orientado a objetos son las clases denominadas SOP (Service-Object Pair), las cuales describen la información y operaciones de una clase de servicio (Sánchez, Rotger y Solarz, 2011), donde cada una de las imágenes manipuladas es definida como una nueva instancia denominada UID, la cual representa la instancia identificadora única de un documento clínico (Subcomité Técnico V3-CDA HL7, 2007), que especifica la codificación y la compresión en los Data Set, tal como se muestra en la Figura 2. Dicha UID contiene todos los atributos obligatorios y opcionales de un IOD. De igual forma debe tener un identificador único denominado OID (Grosso, 1983).

Respecto al "IOD Enhanced", corresponde a una mejora de los objetos IOD, debido a que soporta series más grandes, teniendo en cuenta que una serie es una representación de información en un determinado instante, por ejemplo la información del paciente.

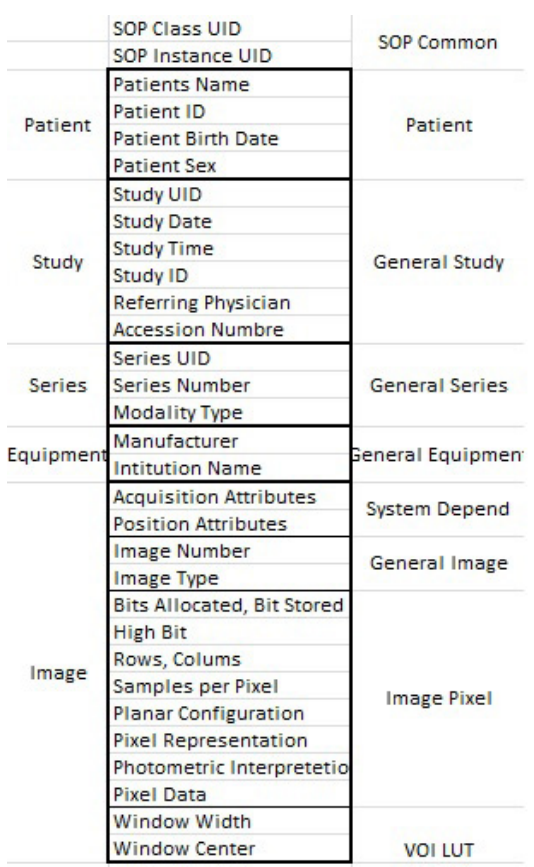

Figura 3. Ejemplo de un IOD de imagen compuesto Fuente: adaptado de Herranz (2003)
En la Tabla 1 se presenta la estructura modular de la mejora representada por cada uno de los UID de los objetos IOD; así mismo, a continuación se explica a nivel general el significado e importancia de cada uno de estos módulos:

- Patient Module: especifica los atributos de los pacientes que describen e identifican al paciente como un sujeto de un estudio de tipo diagnóstico. Dichos atributos son necesarios para la interpretación del diagnóstico a partir de la imagen, y son comunes para todos los estudios llevados a cabo para dicho paciente (DICOM RT Suplement, 1997).

- Clinical Trial Subject Module: Contiene los atributos que identifican al paciente con el tipo de asunto como ensayo clínico (National Electrical Manufacturers Association, 2003).

- General Study Module: especifica los atributos que describen e identifican los estudios realizados a un paciente (National Electrical Manufacturers Association, 1998).

- Patient Study Module: define los atributos que proporcionan la información acerca del tiempo en el cual un estudio fue realizado a un paciente.

- Clinical Trial Study Module: contiene los atributos que identifican un estudio y el contexto de las pruebas clínicas (DICOM Standards Committee, 2004).

- SR Document Series Module: define los atributos para el documento SR basado en la arquitectura del documento a partir de CDA. Una serie de documentos SR puede contener cualquier número de documentos (DICOM Standards Committee, 1999).

- Clinical Trial Series Module: contiene los atributos que identifican el contexto de una serie de pruebas clínicas.

- General Equipment Module: especifica los atributos que identifican y describen los equipos que intervienen en un procedimiento o las series de las instancias que lo componen (DICOM Standards Committee, 2004). 
- $\quad$ SR Document General Module: el documento general SR define los atributos generales de una instancia de documento, donde se identifican y se plantea un contexto a los documentos, como por ejemplos los tipos de reportes (DICOM Standards Committe, 1999).

- SR Document Content Module: los atributos en este módulo permiten la transmisión de la información de un documento SR (DICOM Standards Committe, 1999).

- SOP Common Module: define los atributos que se requieren para el buen funcionamiento y para las identificaciones de las instancias asociadas a las clases SOP (DICOM Standards Committe, 2002).

Tabla 1. Módulos de los Objetos IOD del DICOM mejorado

\begin{tabular}{|llll|}
\hline \multirow{2}{*}{ IE } & \multicolumn{1}{c}{ Module } & Reference & Usage \\
\hline \multirow{2}{*}{ Patient } & Patient & C.7.1.1 & $\mathrm{M}$ \\
\cline { 2 - 4 } & Clinical Trial Subject & C.7.1.3 & $\mathrm{U}$ \\
\hline \multirow{3}{*}{ Study } & General Study & $\mathrm{C} .7 .2 .1$ & $\mathrm{M}$ \\
\cline { 2 - 4 } & Patient Study & $\mathrm{C} .7 .2 .2$ & $\mathrm{U}$ \\
\cline { 2 - 4 } & Clinical Trial Study & $\mathrm{C} .7 .2 .3$ & $\mathrm{U}$ \\
\hline \multirow{2}{*}{ Series } & SR Document Series & $\mathrm{C} .17 .1$ & $\mathrm{M}$ \\
\cline { 2 - 4 } & Clinical Trial Series & $\mathrm{C} .7 .3 .2$ & $\mathrm{U}$ \\
\hline \multirow{2}{*}{ Equipment } & General Equipment & $\mathrm{C} .7 .5 .1$ & $\mathrm{M}$ \\
\hline \multirow{3}{*}{ Document } & SR Document General & $\mathrm{C} .17 .2$ & $\mathrm{M}$ \\
\cline { 2 - 4 } & SR Document Content & $\mathrm{C} .17 .3$ & $\mathrm{M}$ \\
\cline { 2 - 4 } & SOP Common & $\mathrm{C} .12 .1$ & $\mathrm{M}$ \\
\hline
\end{tabular}

Fuente: adaptado de Barberis e Isoardi (2009)

\section{Librería DCM4CHE2}

Para efectuar las transformaciones necesarias, primero es conveniente asumir la interacción que se aplicará en los objetos y data elements del archivo DICOM, por lo que se requiere realizar un procesamiento de la información de los IOD a través de la manipulación de una serie de librerías diseñadas para tal finalidad, dependiendo del lenguaje de programación del cual se vaya a hacer uso (Montoya, 2009).

De acuerdo con lo expuesto por Sánchez et al. (2011), quienes propusieron un estudio y comparación de herramientas de conversión de objetos IOD a partir de archivos de tipo DICOM, es posible un análisis de librerías DICOM (Tabla 2).

A partir del estudio citado, se decidió utilizar como librería de transformación DICOM toolkit (dcm4che2), que es una colección de aplicaciones y utilidades de código abierto para la asistencia médica de TI desarrolladas en el lenguaje de programación Java sobre JDK 1.4 y versiones posteriores.

La suite dcm4che2 de aplicaciones (toolkit DICOM, archivo, etc.) es utilizada en todo el mundo por los proveedores de salud, proyectos de investigación, aplicaciones de código abierto, aplicaciones comerciales, entre otros.

Tabla 2. Análisis de librerías DICOM

\begin{tabular}{|llll|}
\hline Librería & Plataforma/Lenguaje & Formato entrada & Formato salida \\
\hline $\begin{array}{l}\text { Dcm4che2 } \\
\text { DICOM Toolkit }\end{array}$ & Multiplataforma/ Java, XML & DICOM, JPG, txt, pdf, XML & DICOM, txt, XML \\
\hline DCMTK & W,L,M/C++ & DICOM & DICOM \\
\hline Dicom3tools & W,L,M/Linux & DICOM, formatos propietarios & DICOM \\
\hline GDCM & W/C++ & RAW, JPEG, RLE & DICOM, XML \\
\hline Plugin Clear Canvas & W/C\# & PNG, JPEG, BMP & DICOM \\
\hline XMedCon & W,L,M/C & PNG, NEMA, DICOM, Interfile & $\begin{array}{l}\text { PNG, NEMA, DICOM, } \\
\text { Interfile }\end{array}$ \\
\hline
\end{tabular}

Fuente: tomado de Sánchez et al. (2011) 


\begin{tabular}{l|l|l|l}
\hline & DCMTK & DCM4CHEE & CONQUEST \\
\hline $\begin{array}{l}\text { Documentation } \\
\text { Maintainability }\end{array}$ & High & Hotenghensive & Poor \\
\hline $\begin{array}{l}\text { Programming } \\
\text { Language }\end{array}$ & C/C++ & Java, XML & Cower \\
\hline Extendibility & $\begin{array}{l}\text { Add new SOP's, } \\
\text { requires modify } \\
\text { and rebuild the } \\
\text { source code }\end{array}$ & $\begin{array}{l}\text { Add new SOP's } \\
\text { don't always } \\
\text { require modify } \\
\text { the source } \\
\text { code. }\end{array}$ & $\begin{array}{l}\text { Add new SOP's } \\
\text { requires modify } \\
\text { and rebuild the } \\
\text { source code }\end{array}$ \\
\hline $\begin{array}{l}\text { Operating } \\
\text { Systems }\end{array}$ & $\begin{array}{l}\text { UNIX, Linux, } \\
\text { Windows }\end{array}$ & Multiplatform & Windows, UNIX \\
\hline $\begin{array}{l}\text { Client/Server } \\
\text { PACS Model }\end{array}$ & $\begin{array}{l}\text { As Server: } \\
\text { Modality Worklist } \\
\text { and Storage }\end{array}$ & $\begin{array}{l}\text { Provides } \\
\text { complete } \\
\text { support }\end{array}$ & $\begin{array}{l}\text { As Server: } \\
\text { Modality Worklist } \\
\text { and Storage }\end{array}$ \\
\hline $\begin{array}{l}\text { IHE Integration } \\
\text { Profiles }\end{array}$ & None & Several & $\begin{array}{l}\text { None, but } \\
\text { support some } \\
\text { HL7 messages }\end{array}$ \\
\hline DICOM Services & $\begin{array}{l}\text { No Hanging } \\
\text { Protocol support }\end{array}$ & $\begin{array}{l}\text { Hanging } \\
\text { Protocol, } \\
\text { Storage } \\
\text { Commitment, } \\
\text { Basic Worklist }\end{array}$ & $\begin{array}{l}\text { No Hanging } \\
\text { Protocol support }\end{array}$ \\
\hline $\begin{array}{l}\text { DICOM IOD's } \\
\text { (Image Types) }\end{array}$ & $\begin{array}{l}\text { US, CT, MR, SC, } \\
\text { DX, XA, VL, RT }\end{array}$ & $\begin{array}{l}\text { US, CT, MR, } \\
\text { SC, DX, XA, VL, } \\
\text { RT }\end{array}$ & $\begin{array}{l}\text { US, CT, MR, SC, } \\
\text { DX, XA }\end{array}$ \\
\hline
\end{tabular}

Figura 4. Evaluación de frameworks DICOM de tipo open source

Fuente: tomado de Vázquez, Bohn, Gessat y Burgert (2006)

\section{Transformación a XML}

Para realizar la transformación a XML (Ruiz y Trujillo, 2008) fue necesario crear un escenario donde la lectura de información de cada uno de los atributos provenientes de los archivos DICOM correspondientes a algún procedimiento médico utilizando algún dispositivo biomédico.

Debido a las tecnologías involucradas se determinó como ambiente de desarrollo el definido en la Tabla 3.

Tabla 3. Ambiente de desarrollo propuesto

\begin{tabular}{|ll|}
\hline Versión de Java & JDK 1.6 \\
\hline IDE de desarrollo & Netbeans 7.4 \\
\hline Versión de librería dcm4che2 & 2.0 .25 \\
\hline Librerías dcm4che utilizadas & $\begin{array}{l}\text { • dcm4che-imageio } \\
\text { dcm4che-core } \\
\text { B dcm4che-xml }\end{array}$ \\
\hline Base de datos & MySQL \\
\hline
\end{tabular}

Fuente: elaboración propia.

Seguidamente se tomó como muestra una serie de imágenes, del mismo tipo de las planteadas en la Figura 5, correspondientes a una ecografía de una paciente clínica, para realizar la extracción de información.

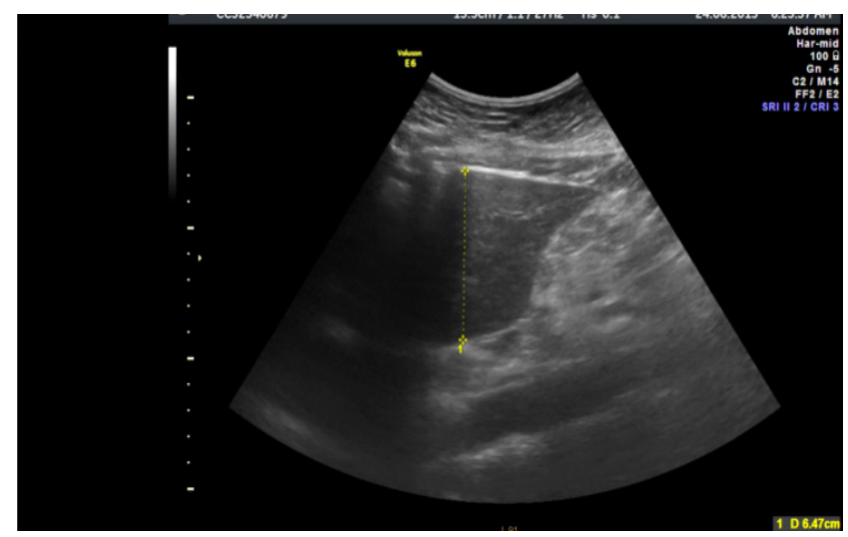

Figura 5. Imagen de muestra, correspondiente a una ecografía

Teniendo en cuenta lo planteado, y explorando la librería elegida, desarrollada dentro del marco definido por el paradigma de orientación a objetos, se planteó el modelo de la Figura 6, correspondiente a una representación conceptual en lenguaje de modelado UML de la capa lógica de una aplicación en Java, con una serie de métodos que permitieron la lectura, manipulación y transformación de la información.

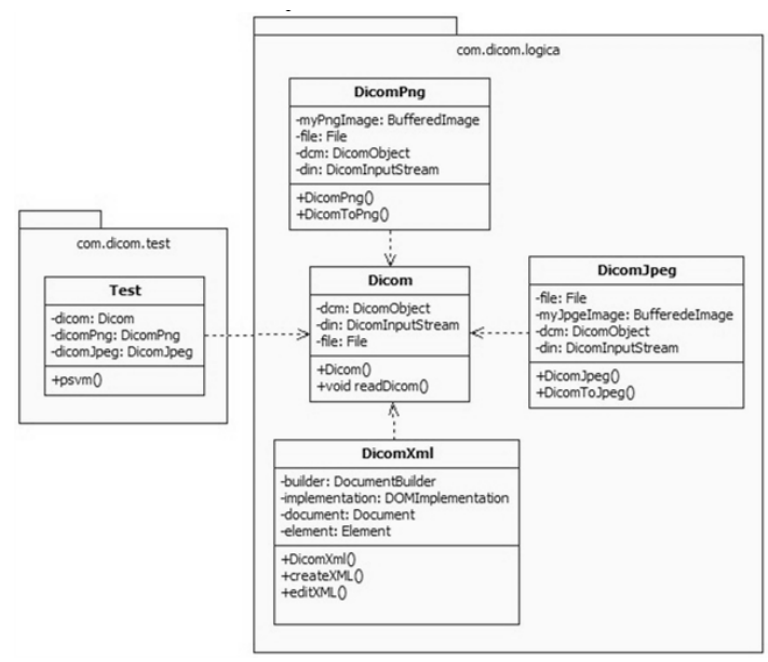

Figura 6. Modelo de lectura y transformación de información DICOM

Fuente: elaboración propia 
Una vez instanciado el UID (Identificador Único), de cada IOD, de las clase SOP, es posible acceder a los atributos (tags) de cada objeto, de esta forma a cada uno de estos atributos es posible realizarle un casting a objetos de tipo String, tal como se muestra en la Figura 7.

Posteriormente, es necesario crear un archivo XML para convertir la información contenida en el DICOM a formato XML estándar, para lo cual se procedió a utilizar la librería JDOM (Mclaughlin, 2001), que permite leer, escribir, crear y manipular archivos XML de forma intuitiva.

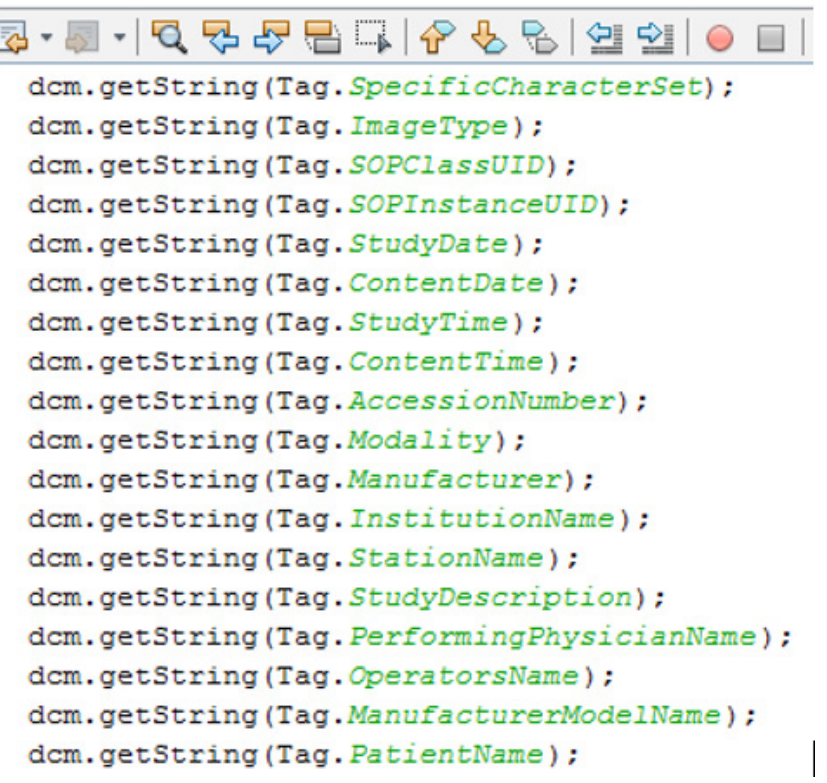

Figura 7. Casting de UID a String Fuente: elaboración propia

Java, utilizado como lenguaje de programación, permitió trabajar bajo lo especificado por el paradigma de programación orientado objetos; a partir de cada una de las instancias correspondientes a una imagen de tipo DICOM fue posible extraer la información concerniente a los objetos UID y realizar un casting a objetos de tipo String, tal como lo muestra la Figura 7.

Parseado el objeto de la imagen DICOM, fue necesario crear una serie de nodos, cada uno de los cuales corresponde a los tags que mapean la información de los objetos UID en información
$\mathrm{XML}$, y generan la información proveniente de los archivos DICOM en archivos $\mathrm{XML}$, tal como se muestra en la Figura 8.

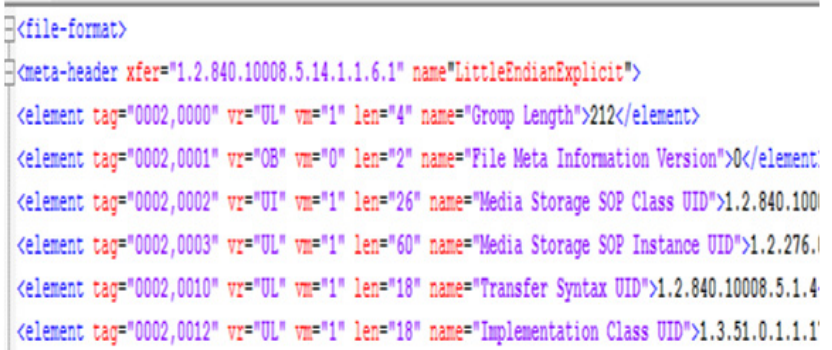

Figura 8. Archivo XML resultante

Fuente: elaboración propia

Dicha información se garantiza al utilizar un visor DICOM, en este caso de marca Samsung, que proporciona la información correspondiente a la imagen DICOM utilizada, como se muestra en la Figura 9.

\section{Transformación A Formatos JPEG Y PNG}

De igual forma, es posible convertir un archivo de tipo DICOM casi siempre con extensión .dcm a un formato más familiar en diferentes sistemas de información, por ejemplo, JPEG o PNG (Gaziandia et al., 2011).

Para tal fin, la librería dcm4che cuenta con utilidades que realizan esta conversión —al igual que en el caso anterior - a archivos XML, por lo cual se optó por realizar un procesamiento de la información a través de una librería que fuese concebida exclusivamente para realizar la manipulación de información sobre objetos Java; la librería escogida fue DOM (que utiliza una estructura de nodos para creación y lectura de archivos XML), la cual a partir de una serie de nodos permite definir una serie de elementos para la creación y lectura de archivos XML.

De igual forma, para el tratamiento de información es posible crear un objeto estático como una imagen con los métodos de la librería dcm4che: se instancia una series de buffers de lectura y 


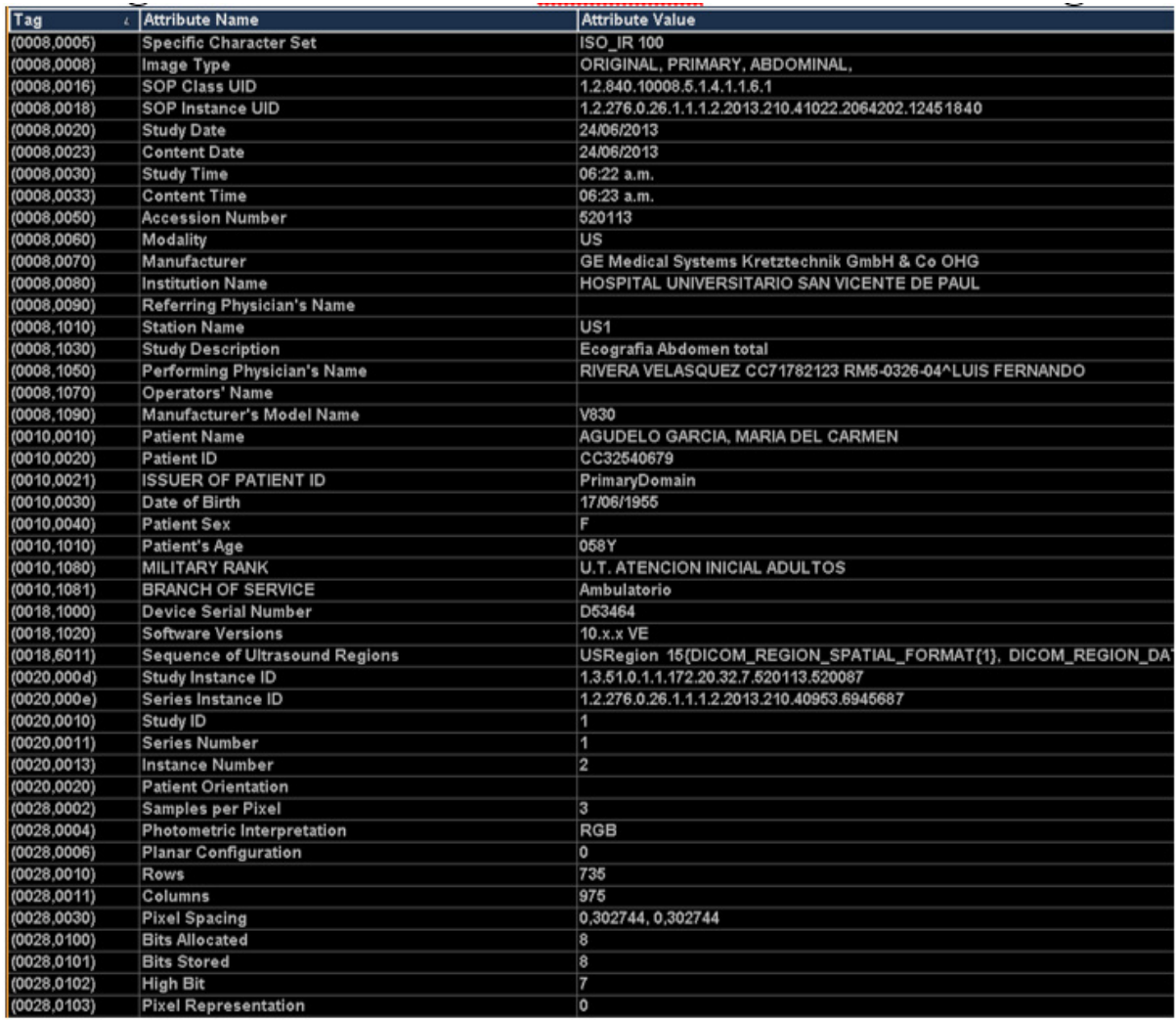

Figura 9. Información DICOM con visor Samsung Fuente: Elaboración propia mediante un Visor Samsung

de escritura para generar un objeto con extensión PNG o JPEG, y se referencian las imágenes que se van a convertir. Como resultado se tiene el JPEG o PNG de la imagen original de tipo DICOM (Gaziandia et al, 2011).

La Figura 10 muestra un método para convertir, encargado de la construcción de un archivo de tipo JPG a partir de la información parseada anteriormente. Al igual que para cualquier archivo de este tipo, fue necesario crear una serie de buffers encargados de almacenar de forma estática la información concerniente, para luego persistirla en una ruta absoluta.

\section{Conclusiones}

El proceso de transformación de la información proveniente de las imágenes de tipo DICOM puede cambiar dependiendo de las tecnologías que se vayan a utilizar, así mismo, las librerías necesarias dependen, en alta medida, del paradigma y del lenguaje de programación que se vaya a implementar.

Dentro de las librerías analizadas para el tratamiento de información DICOM, una de las mejores opciones es dcm4che DICOM toolkit, debido a la diversidad de utilidades y ventajas en comparación a otros frameworks open source presentes en el mercado.

A partir del esquema propuesto, es posible plantear un modelo de interoperabilidad de servicios DICOM basado en las utilidades de las librerías escogidas en estructuras que posibilita la transferencia de información a través de $X M L$, entre los diferentes sistemas, servicios y demás recursos que necesiten información de tipo DICOM, proveniente de diferentes servicios o procedimientos médicos. 


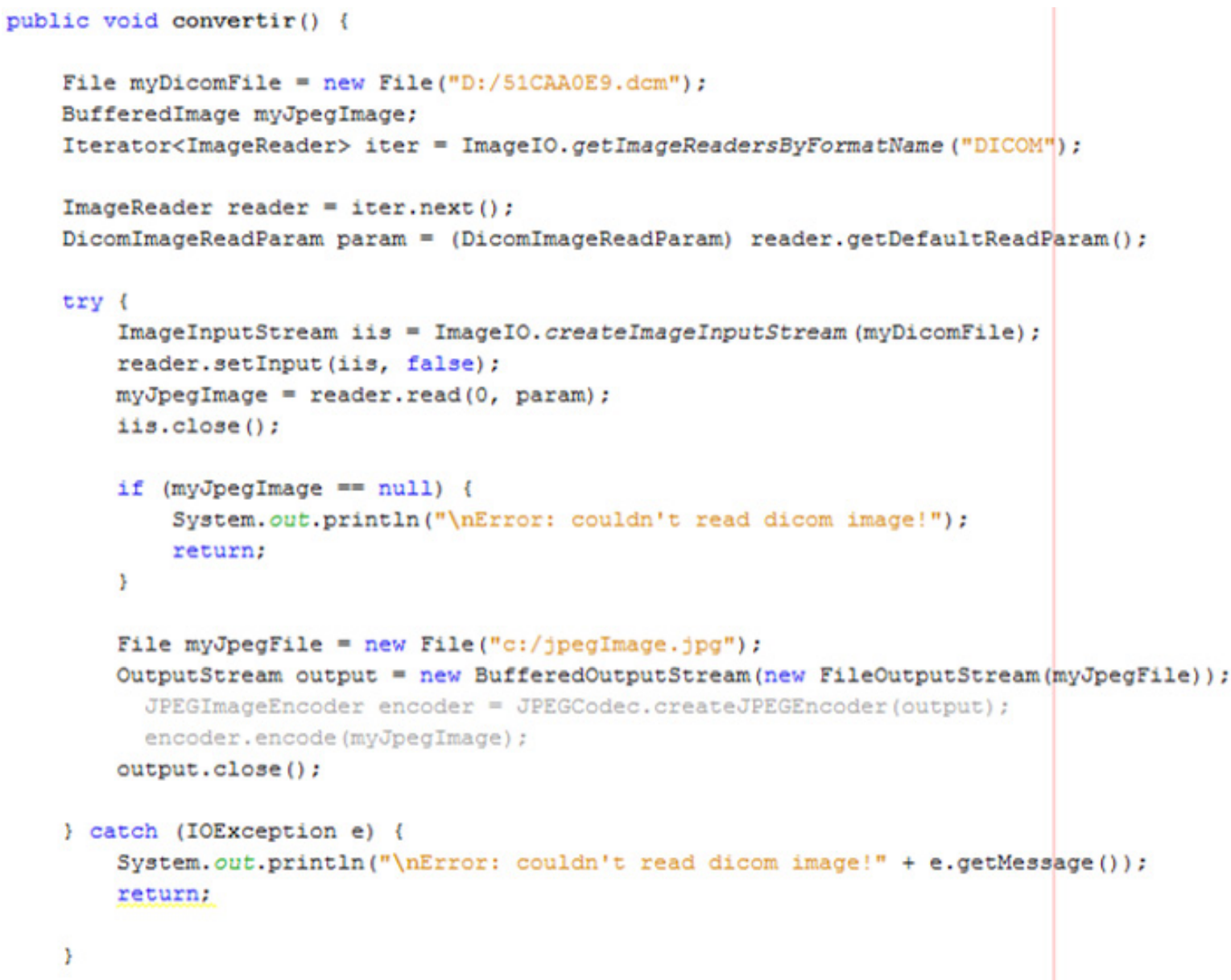

Figura 10. Implementación en Java de conversión de DICOM a JPG

Fuente: elaboración propia

\section{Referencias}

Barberis L. e Isoardi R. (2009). "Aplicación de un sistema automático de procesamiento de imágenes médicas basada en estándares".

DICOM RT Suplement. (June 1997). Digital Imaging and Communications in Medicine (DICOM). Supplement 11: Radiotherapy Objects.

DICOM Standards Committe. (1999). Digital Imaging and Communications in Medicine (DICOM). Supplement 23: Structured Reporting Storage SOP Classes.

DICOM Standards Committee. (2002). Digital Imaging and Communications in Medicine (DICOM). Table of Contents, 49.
DICOM Standards Committee. (2004). Digital Imaging and Communications in Medicine (DICOM). Supplement 104: DICOM Encapsulation of PDF Documents.

Gaziandia M. A., Arriezu L. S., Navarro J. A., Analyzer F., De Carl I. I. and El Z., "Dicomización e integración de un equipo de imagen médica con un PACS: caso práctico de un equipo de Campimetría ." 2011.

Grosso, R. (1983). "Nociones básicas sobre comunicación y almacenamiento de imágenes en DICOM," 1-25.

Grupo PAS - Universidad de Deusto (2010). "Estándar y Protocolo de Imágenes Medicas DICOM", 1-17. 
Herranz, F. B. (2003). "Desarrollo de aplicaciones DICOM para la gestión de imágenes biomédicas".

Mclaughlin, B. (2001). "Java + XML = JDOM".

Montoya, A. (2009). "Diseño e implementación de un sistema telemático para equipos médicos usando el protocolo de comunicación DICOM".

National Electrical Manufacturers Association. (1998). Digital Imaging and Communications in Medicine (DICOM) Supplement 17: Modality Performed Procedure Step.

National Electrical Manufacturers Association. (January 2003). "Digital Imaging and Communications in Medicine (DICOM) Supplement 70: Clinical Trial Identification Scope and Field of Application," 1-11.

Parisot, C. (June 2003). "The Basic Structure of DICOM".
Pianykh, O. S., (2008). Digital Imaging and Communications in Medicine (DICOM).

Rosslyn, S. (2004). "Digital Imaging and Communications in Medicine (DICOM) Part 8: Network Communication Support for Message Exchange".

Ruiz C. y Trujillo A. (2007). "Aproximación a la representación en XML de objetos DICOM para fotografía médica digital," 147-163.

Sánchez, M., Rotger V. y Solarz I. (2011). "DICOM de ECG para Telemedicina," 1-10.

Subcomité Técnico V3-CDA HL7 (2007). Guía para el desarrollo de documentos CDA. Recuperado de http://www.hl7spain.org/ documents/comTec/cda/GuiaElementosMinimosCDA.pdf

Vázquez A., Bohn S., Gessat M. y Burgert O. (2006). "Evaluation of Open Source DICOM Frameworks," p. 9712012. 
